\title{
Alimentation de rue à Bamako au Mali: problématique et approches d'intervention
}

\author{
Mohamed Ag Bendech, \\ Bureau Régional de la FAO pour l'Afrique, Rue Gamel Abdul Nasser, Boite Postale GP 1628, Accra, Ghana.
}

\begin{abstract}
Je remercie le Pr Akory Ag Iknane, Directeur de l'Agence en charge de la Sécurité Sanitaire des Aliments au Mali pour sa revue et ses précieux conseils lors de la finalisation du présent article. Je remercie également le Dr Mahalmoudou Hamadoun du CILSS, Nicolo Giorgia et Richemont Seki de la FAO pour leur engagement dans l'amélioration de l'alimentation de rue en Afrique.
\end{abstract}

\section{Aspects generaux}

Villes et alimentation de rue

En occident, la restauration hors domicile est devenue, grâce au développement de l'industrie agro alimentaire(incluant les Fast- Food), le support de la modernisation alimentaire. Elle constitue de ce fait un axe contribuant à formaliser les comportements (1). Cette modernisation conduit progressivement au développement d'une même logique économique, globalisante et homogénéisante avec des problèmes émergeants de sécurité sanitaire des aliments liés entre autres à l'utilisation des farines animales dans l'alimentation du bétail.

Dans les villes d'Afrique de l'Ouest, le recours aux aliments de rue est devenu de plus en plus courant. Beaucoup plus qu'un phénomène de mode ou de classes, cette forme de restauration est une nécessité pour les citadins particulièrement ceux issus des classes moyennes et pauvres (2). Elle se développe de façon artisanale en créant la rupture avec la logique homogénéisante de l'alimentation en milieu rural. Le nivellement des goûts alimentaires favorisé par l'alimentation familiale à domicile observé en milieu rural s'éloigne progressivement grâce à l'accès individualisé aux aliments de rue. L'extrême dispersion économique et la gamme étendue de l'offre des mets font de ce secteur un atout considérable pour son essor. II s'agit d'un secteur qui innove sans cesse en créant de nouvelles cuisines issues des emprunts entre villes de la sousrégion (Attieké et alloco d'Abidjan, Bissap de Dakar et les pattes de bœuf fumées de Ouagadougou par exemple) et des échanges avec l'occident et l'Asie grâce aux importations (utilisation fréquente du pain, des conserves, du bouillon cube et des condiments par exemple). A travers cette restauration, les cultures culinaires d'origines diverses communiquent librement grâce aux facilités offertes par l'organisation des villes et l'association de deux entités alimentaires : le « local » et « l'importé».

Les services en charge de l'aménagement de l'espace urbain se préoccupent prioritairement de l'occupation des trottoirs, des arrêts de bus, des alentours des marchés et places publiques, de l'élimination des déchets tandis que les municipalités s'interrogent sur l'attitude à adopter face à ce secteur dit informel qui échappe dans une certaine mesure aux règles de l'économie marchande et sur ses relations avec le secteur planifié. De même, les secteurs publics de la santé et du contrôle sanitaire pensent aux risques et avantages de l'alimentation de rue en terme d'hygiène et de sécurité alimentaire et nutritionnelle. Les vendeurs et vendeuses sont plutôt dans une logique du profit, au détriment de la qualité des mets et du service, pour minimiser les risques de famine à leurs familles.

Familles urbaines et alimentation de rue La structure des familles urbaines se réduit en même temps que s'accentue la crise économique qui se caractérise par l'accroissement du chômage et l'absence des programmes sociaux de grande envergure. Le type de famille de la société traditionnelle, élargie, à structure complexe, coexiste avec de modèles centrés sur la famille restreinte. Les réseaux de solidarité sont de plus en plus sélectifs au profit des classes moyennes et favorisées tandis que les échanges alimentaires entre villes et campagnes évoluent en privilégiant ceux de type marchand. Les changements de la structure familiale et ceux liés à l'accès permanent aux aliments de rue n'ont heureusement pas aboutit à la disparition de la notion du repas familial à domicile qui se conserve dans sa structure (type de plats et horaire de repas). La vente des aliments de rue, ne nécessitant pas des investissements de départ importants, permet à toutes les familles urbaines pauvres d'accéder à des revenus. A Bamako, $80 \%$ des familles défavorisées ont au moins un membre engagé dans la vente des aliments de rue contre respectivement $58 \%$ et $14 \%$ des familles moyennes et aisées (3-4). Les vendeuses, souvent chefs de famille, tirent de cette activité un revenu souvent supérieur au salaire minimum. Ce secteur est donc à la fois un facteur de protection de la structure familiale et de régulation de la pauvreté urbaine.

Réduction des Inégalités d'accès à la nourriture et alimentation de rue

La vente des aliments de rue permet d'acquérir un revenu non négligeable qui est réinvesti dans l'alimentation familiale à domicile particulièrement dans les familles urbaines les plus pauvres. De même, c'est grâce à ce secteur que les citadins les plus pauvres accèdent à des aliments riches en micronutriments comme les fruits et légumes et à des aliments industriels enrichis en vitamine A ou susceptibles de l'être à travers par exemple les produits de friture (huiles) et les boissons traditionnelles (sucre).

\section{Offre des aliments de rue}

\section{Caractéristiques des vendeurs/vendeuses}

Les vendeurs/vendeuses des aliments de rue sont à $80 \%$ des femmes de faible niveau d'éducation. A Bamako, $56 \%$ des vendeuses des aliments prêts à être consommés à l'école ont un âge compris entre 21 et 40 ans et $20 \%$ ont moins de 19 ans (3). Selon les pays et 
les villes, il existe plusieurs types de vendeurs/vendeuses d'aliments en fonction du lieu de vente (fixe avec ou sans abri/ambulant), du type et nombre de produits/plats vendus ou encore en fonction du moment de la vente (jour/nuit).

\section{Prix des aliments vendus dans la rue}

La restauration de rue coûte plus chère que celle du domicile (3). Cependant, l'illusion d'une offre alimentaire d'un bon rapport qualité/prix est entretenue sans doute parce que le citadin acquiert à travers ce mode de restauration une autonomie lui permettant de manger ce qu'il veut quand il veut. Cette illusion nous paraît utile car elle contribue à la survie de nombreuses familles urbaines et à la diversification de l'alimentation des groupes vulnérables notamment les enfants d'âge préscolaire.

\section{Qualité hygiénique des aliments de rue et groupes à risque}

Les aliments vendus sur la voie publique sont de qualité douteuse. Des intoxications ont été reportées dans plusieurs pays, à partir des produits à base de lait au Sénégal en 1990 et avec des boissons en inde (2). Les aliments de rue ont été identifiés comme un facteur important dans la propagation de l'épidémie de choléra en Amérique Latine en 1991/92 (2). A Abidjan, une étude portant la salubrité de la glace alimentaire en bloc, de la production à la vente sur les marchés, a montré que, malgré la potabilité de l'eau à la source, les échantillons présentaient une altération de la qualité physicochimique et microbiologique (2). Une étude réalisée au Ghana et publiée dans le bulletin de l'OMS a révélé que certains aliments vendus sur la voie publique (salade, spaghetti, omo tuo et sauce piquante) avaient des niveaux de contamination inacceptables. Au moins 70\% des échantillons d'aliments prélevés contenaient des microbes (5). Outre les faibles connaissances des vendeurs/vendeuses du lien entre Diarrhée et microbes pathogènes, plusieurs facteurs de risque ont été identifiés tout au long de la chaîne d'approvisionnement, préparation, emballage, distribution et environnement de vente/consommation.

Les enfants d'âge préscolaire des familles pauvres sont probablement les plus exposés aux toxi-infections à cause de leur accès quasi-permanent à ces aliments suivis dans une moindre mesure par les écoliers du primaire. Dans un tel contexte, la sécurité sanitaire et la qualité des aliments de rue doivent devenir des enjeux socio-économiques de grande importance.

\section{Demande des aliments de rue}

Consommateurs et consommation des aliments de rue

Les aliments de rue sont consommés par tous quel que soit son statut social, son âge et son sexe. La diversité et le type d'aliments achetés et consommés dépend à la fois de la catégorie socio-économique et de l'âge des sujets. Certaines études révèlent qu'environ $20 \%$ des apports énergétiques quotidiens des individus proviennent de ce mode d'alimentation(3-4). De même, la dépense qu'il représente atteint $20 \%$ de la dépense individuelle quotidienne. A Bamako, l'alimentation de rue représente 19 à $27 \%$ du Budget alimentaire familial (3). L'alimentation de rue offre un débouché aux productions locales peu consommées en famille tels que les fruits et légumes, les boissons traditionnelles et les produits de friture. Elle s'avère un complément nutritionnel indispensable aux sujets de niveaux socio-économiques intermédiaire et défavorisés. En effet, à Bamako, seulement $66 \%$ et $56 \%$ des familles intermédiaires et défavorisées couvrent leurs besoins énergétiques par l'alimentation du domicile (3-4). Les enfants d'âge préscolaires (2-6 ans) des familles pauvres, les plus à risque nutritionnel, ont plus fréquemment recours à ce mode d'alimentation à cause de l'implication de leurs mères dans la vente des aliments de rue. Les écoliers semblent représenter le deuxième groupe le plus exposé à ce mode d'alimentation. Ils disposent quotidiennement de pécule leur permettant d'accéder à une offre variée. $A$ Bamako comme à Cotonou $91 \%$ des écoliers du primaire disposent d'un pécule qui leur permet d'acheter de façon autonome des aliments de rue à l'école (6).

Raisons du recours aux aliments de rue

De multiples raisons expliquent le recours à l'alimentation de rue notamment la contrainte liée à l'activité professionnelle, le plaisir gustatif, le complément alimentaire individualisé et la solitude urbaine touchant surtout les migrants et les célibataires isolés.

\section{Approche d'intervention}

\section{Préalables}

Avant toute intervention tendant à améliorer cette composante, il est important de reconnaître la responsabilité des municipalités dans le contrôle, le soutien et la coordination des actions en lien avec le secteur informel de l'alimentation. II est aussi important de faire émerger une vision commune de tous les acteurs concernés par l'alimentation de rue. Cette vision doit être positive et constructive plutôt que « répressive ».

\section{Quelques axes d'intervention}

Les mesures destinées à améliorer le secteur de l'alimentation de rue doivent être multidimensionnelles. Une bonne analyse de situation est souhaitée comme point de départ à toute démarche d'intervention car ce secteur se développe de façon diverse selon les pays et les villes. Les principales actions doivent porter sur l'amélioration des revenus des vendeurs/vendeuses par l'accès au micro-crédit, l'amélioration de la qualité hygiénique et nutritionnelle des aliments et la protection du consommateur. La question du cadre légal pour le secteur informel de l'alimentation doit être appréhendée avec beaucoup de souplesse et d'innovations pour éviter la rupture de l'équilibre actuel. Tous ces axes passent par une information et parfois la formation des partenaires (décideurs, techniciens, vendeurs/vendeuses, consommateurs, agents municipaux: service d'hygiène et de contrôle sanitaire, 
de police et de voirie, associations des consommateurs et diverses associations urbaines et ONGs). La FAO a déjà documenté plusieurs expériences de soutien à ce secteur, notamment dans l'amélioration de la qualité hygiénique des aliments de rue et les formations des vendeuses/vendeurs conduites dans plusieurs grandes villes d'Afrique de l'Ouest dont Bamako et Ouagadougou (7). Le Comité Inter-état de lutte Contre la sécheresse au Sahel (CILSS) et la FAO travailleront ensemble dans trois pays du Sahel (Cote d'Ivoire, Mali et Tchad) pour améliorer les capacités des acteurs urbains dans la vente des aliments de rue de qualité hygiénique et nutritionnelle améliorée (8). Au cours de la mise en œuvre de ladite action, le CILSS et la FAO coopéreront étroitement avec les parties prenantes dans les pays concernés en vue d'établir dans chacun d'eux un plan d'action détaillé et assorti d'une estimation des coûts prenant en compte les leçons apprises afin d'étendre le projet dans les villes ciblées et d'autres petites villes tout en cherchant les opportunités de partenariat et de financement.

\section{Conclusion}

L'analyse de cette composante de l'alimentation urbaine montre que les logiques des acteurs qui se dessinent sont parfois contradictoires. II est important d'avoir une démarche pragmatique vis à vis du secteur de l'alimentation de rue en prenant en compte plusieurs de ses dimensions pour assurer de façon harmonieuse son intégration au développement global de la ville. Pour cela, il est suggéré d'avoir un système régulier de sa documentation pour maîtriser les évolutions, renseigner la décision dans les municipalités et développer des actions concrètes et adaptées au contexte de vie de l'ensemble des partenaires urbains. C'est dans ce cadre que s'inscrit le travail en cours de la FAO sur l'amélioration des conditions de la vente des aliments de rue dans les villes en Afrique.

\section{Références bibliographiques}

1) Ariès $P$. La fin des mangeurs. Editions Desclée de Brouwer, Paris 1997.

2) Chauliac $M$ et al. Villes et alimentation: la consommation dans les rues. Rapport CIE 1993.

3) $\mathrm{Ag}$ Bendech $\mathbf{M}$ et al. Les enjeux de la consommation alimentaire en milieu urbain à Bamako. Santé Publique 2000, volume 12, no 1, pp. 43-65.

4) Ag Bendech M., Chauliac M et Malvy D. Assessment of dietary intake at home and outside the home in Bamako (Mali). Ecology of Food and Nutrition $1998 ; 37$ : 135-162

5) Mensah P et al. Street foods in Accra, Ghana : how safe are they? Bull WHO 2002, 80 (7): 546-554.

6) Chauliac M, Monnier T, Ag Bendech M. Les écoliers de Bamako et l'alimentation de rue. Cahiers Santé 1994 ; 4 :413-23.

7) Nicolo Giorgia and Mohamed Ag Bendech Street food vending in West Africa: Potential and challenges, FAO Regional Office for Africa, 2012 (soumis pour publication).

8) FAO/CILSS. Ensuring the provision of safe and quality street food for better public health in Cote D'Ivoire, Mali and Chad. TCP/RAF 3406, Project 2013. 